\title{
Hydrodynamics within the Electric Double Layer on slipping surfaces
}

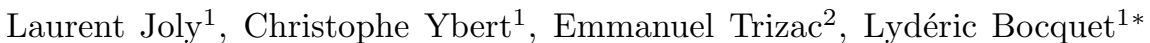 \\ 1 Laboratoire P.M.C.N., UMR CNRS 5586, Université Lyon I, 69622 Villeurbanne, France \\ 2 L.P.T.M.S, UMR CNRS 8626, Bâtiment 100, Université Paris XI, 91405 Orsay, France
}

(Dated: November 7, 2018)

\begin{abstract}
We show, using extensive Molecular Dynamics simulations, that the dynamics of the electric double layer (EDL) is very much dependent on the wettability of the charged surface on which the EDL develops. For a wetting surface, the dynamics, characterized by the so-called Zeta potential, is mainly controlled by the electric properties of the surface, and our work provides a clear interpretation for the traditionally introduced immobile Stern layer. In contrast, the immobile layer disappears for non-wetting surfaces and the Zeta potential deduced from electrokinetic effects is considerably amplified by the existence of a slippage at the solid substrate.
\end{abstract}

PACS numbers: 68.15+e,47.45.Gx,82.45.-h

The electric double layer (EDL) is a central concept in the understanding of the static and dynamical properties of charged colloidal systems. This notion was introduced in the early works of Gouy, Debye and Hückel [1] to describe the distribution of micro-ions close to a charged colloidal surface. The EDL width determines the electric interaction range between macromolecules and therefore controls the static phase behaviour of these systems. On the other hand, at the dynamical level the EDL is at the origin of numerous electrokinetic effects [1] : electrophoresis, electro-osmosis, streaming current or potential, etc. Because these various phenomena are governed by the surface of the sample via the EDL, they provide smart and particularly efficient ways to drive or manipulate flows in micro-fluidic devices 2, 3], where surface effects become predominant.

The extension of the EDL is typically on the order of a few nanometers and electrokinetic phenomena therefore probe the nanorheology of the solvent+ions system at the charged surface. This raises therefore some doubts about the validity of continuum approaches to describe the dynamics at such scales. These doubts are particularly relevant concerning the traditional description of the EDL dynamics, which relies both on the mean-field Poisson-Boltzmann theory of the micro-ion clouds, but also on continuum hydrodynamics for the flow fields 1 . These two aspects are embodied in the so-called Zeta potential, denoted $\zeta$, which is traditionally defined as the electric potential $V\left(z_{s}\right)$ computed at the surface of shear $z_{s}$, where the fluid velocity vanishes $[1,4,[5,6]$. This definition of $\zeta$ however relies on the somewhat uncontrolled assumption of no-slip boundary condition of the solvent at the solid surface [7].

This assumption has been critically revisited in the last years. Indeed, a lot of progress has been made recently in the understanding of the rheology of fluids at small scales, thanks in particular to computer simulations, such as Molecular Dynamics (see eg [8] and references therein), but mainly to the development of new experimental techniques, such as Surface Force Appara- tus (SFA) or Atomic Force Microscope (AFM) [9, 10]. The conclusions emerging from these studies are that, while continuum hydrodynamics are found surprisingly to remain valid up to very small length scales, the noslip boundary condition (BC) for the fluid velocity at the solid surface may be violated in many situations (see 7, 8, 9, 10] and refs. therein). Moreover, this violation of the usual no-slip BC is found to be controlled by the wetting properties of the fluid on the solid surface : while the no-slip BC is fulfilled on hydrophilic surfaces, a finite velocity slip is measured on hydrophobic surfaces [8, 10].

In this Letter we show using Molecular Dynamics (MD) simulations that a finite slip effect for the solvent at a charged surface considerably enhances the measured electrokinetic effects. This results in an enhanced $\zeta$ potential, the origin of which lies in the dynamics of the solvent at the surface: the flow-induced current, driven by the convection of micro-ions in the EDL, is much larger in the presence of velocity slip at the surface than for the corresponding situation with a no-slip BC. Moreover, while an immobile Stern layer develops close to the charged surface on hydrophilic samples, and does not contribute to electrokinetic effects, this layer is absent on hydrophobic surfaces and does contribute in a significant way to charge transport.

We first precise our microscopic model and some details of the simulation procedure. The fluid system (solvent and micro-ions) is confined between two parallel solid substrates, composed of individual atoms fixed on a fcc lattice. The solvent and solid substrate particles interact via Lennard-Jones (LJ) potentials,

$$
v_{i j}(r)=4 \epsilon\left[\left(\frac{\sigma}{r}\right)^{12}-c_{i j}\left(\frac{\sigma}{r}\right)^{6}\right]
$$

with identical interaction energies $\epsilon$ and molecular diameters $\sigma$. The tuning parameters $c_{i j}$ allow to adjust the wetting properties of the fluid on the substrate [8] : for a given fluid-fluid cohesion $c_{F F}$, the substrate exhibits a "hydrophilic" behavior for large fluid-solid cohesivity, $c_{F S}$, and a "hydrophobic" behavior for small 
$c_{F S}$. Here, the wetting (respectively non-wetting) situation is typically achieved by taking $c_{F S}=1$ (resp. 0.5) for a fixed $c_{F F}=1.2$. This leads to a contact angle $\theta$ of a liquid droplet on the substrate, measured in the simulations equal to $80^{\circ}$ (resp. $140^{\circ}$ ) for a temperature $k_{B} T / \epsilon=1$ (we refer to [8] for an exhaustive discussion on this point). On the other hand, micro-ions interact both through LJ potentials, as described in Eq. (1), and Coulomb potential in a medium with dielectric permittivity $\epsilon_{d}\left[v_{\alpha \beta}(r)=k_{B} T q_{\alpha} q_{\beta} \ell_{B} / r\right.$ where $q_{\alpha}, q_{\beta}$ are the valences of the interacting charges, and $\ell_{B}=e^{2} /\left(4 \pi \epsilon_{d} k_{B} T\right)$ is the Bjerrum length, $e$ denoting the elementary charge]. In water at room temperature $\ell_{B}=0.7 \mathrm{~nm}$. We shall choose in our simulations $\ell_{B}=\sigma$. Ions also interact directly with the surface atoms via the same LJ potential as the solvent (i.e. same $c_{F S}$ ). Micro-ions and solvent particles have the same size $\sigma$ (in Eq. (1)). Wall atoms are organized into five layers of a fcc solid (100 direction) in both walls. For each wall, the first layer only, in contact with the fluid, is charged. The corresponding $N_{w}$ atoms bare a discrete charge, with valency $q_{\text {wall }}=-Z / N_{w}$ so that each wall bears a negative net charge $-Z e$. The solvent contains $Z$ monovalent counter-ions, to which $N_{s}=N_{+}+N_{-}$salt ions are added, all with valence one. Global electroneutrality is enforced by imposing $N_{+}=N_{-}$. The systems simulated are generally made up of $10^{4}$ atoms. A typical solvent density is $\rho_{f} \sigma^{3} \sim 1$, while the concentration of micro-ions $\rho_{s}=N_{ \pm} / V$ is varied between $\rho_{s} \sigma^{3}=5.10^{-3}$ and $\rho_{s} \sigma^{3}=0.16$ (with $V$ the total volume of the sample). This corresponds typically to an ionic strength varying between $10^{-2} M$ and $1 M$. The corresponding Debye screening length (see below) is accordingly of the order of a few Bjerrum lengths. The charge per unit surface on the wall is $\Sigma=-0.2 e . \sigma^{-2}$, associated to a Gouy-Chapman length $\lambda=\left(2 \pi l_{\mathrm{B}} \Sigma\right)^{-1}=0.8 \sigma$. Using a typical value $\sigma=5 \AA$, this corresponds roughly to $-0.13 C . m^{-2}$. Periodic boundary conditions are applied in the $x$ and $y$ directions, with $L_{x}=L_{y}=16 \sigma$ while the distance between the walls is $L_{z}=20.8 \sigma$. Ewald sums are used to compute Coulombic interactions (assuming a periodicity in the $z$ direction with a box size of $112 \sigma$, much larger than the wall to wall distance) [11]. Lennard-Jones units are used in the following [distance $\sigma$, time $\left.\tau=\left(m \sigma^{2} / \epsilon\right)^{1 / 2}\right]$. Temperature was kept constant by applying a Hoover thermostat to the $y$ degrees of freedom, i.e. in the direction perpendicular to the flow and confinement.

Our model therefore includes the discrete nature of the solvent and charges and a tuning wettability of the surface, whereas these effects are usually neglected in the traditional description of electrokinetic phenomena. Note that we chose to describe the charge interaction at the level of an effective dielectric media (with dielectric permittivity $\epsilon_{d}$ ). This simplifying assumption -that could be relaxed using a more realistic model for the solvent [5]- allows to investigate specifically the generic in-

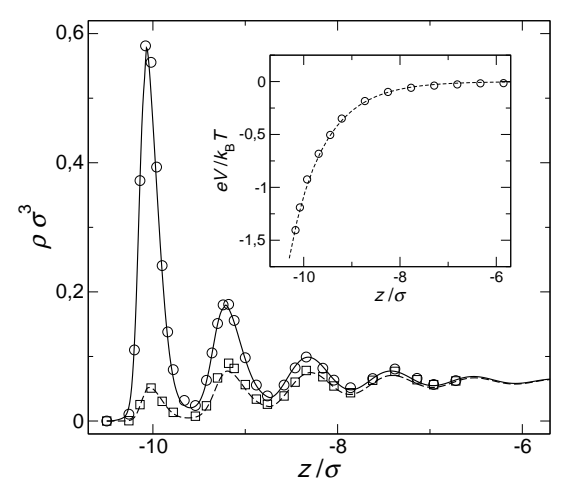

FIG. 1: Micro-ionic density profiles, averaged over the $x y$ directions $\left(\rho_{s} \sigma^{3}=0.07\right.$, wetting case). Symbols : MD simulations results for the counter-ions (o) and co-ions ( $\square$ ); Solid and dashed lines : corresponding predictions of the modified PB description (see text). Inset: Electrostatic potential. The wall is located at $z_{w}=-10.8 \sigma$. Symbols (o): MD simulation results calculated from Poisson's equation and micro-ions profiles; Dashed line : bare PB prediction (see text).

terplay between slip effects and electric behavior, which is the main focus of this work. We have moreover explored situations beyond the ones presented here (with or without salt, using different $c_{F S}$ for the ions and the solvent, and various Bjerrum lengths $\ell_{B}$ ), leaving the present conclusions unaffected.

We now turn to the simulation results. We first focus on the equilibrium properties of the EDL. In Fig. 11 we show typical density profiles. The latter are found to exhibit important structuration effects close to the charged surface and thus depart strongly from the Poisson-Boltzmann (PB) prediction [1]. However, the oscillations in the micro-ions profiles originate in the structuration in the solvent itself, and such an effect can be captured by a modified PB description. Indeed, due to the presence of the solvent, micro-ions not only organize due to electric interactions (which corresponds to the usual PB description) but also due to the effective external field associated with the structuration in the solvent [12], defined as $V_{\text {ext }}(z)=-k_{B} T \log \left[\rho_{f}(z) / \rho_{f}\right]$, with $\rho_{f}(z)$ the solvent density profile and $\rho_{f}$ its bulk value. The micro-ions density profiles $\rho_{ \pm}(z)$ correspondingly obey a modified Boltzmann equilibrium :

$$
\rho_{ \pm}(z) \propto e^{\beta\left(\mp e V(z)-V_{e x t}(z)\right)} \propto \rho_{f}(z) e^{\mp \beta e V(z)}
$$

with $\beta=1 / k_{B} T$ and $V(z)$ the electrostatic potential. Actually, such a relationship emerges naturally from a simple Density Functional Theory, in which the discrete nature of both solvent and charged atoms is taken into account exactly, while the standard mean-field PB free energy is assumed for the electrostatic part. Using Poisson's equation, the electrostatic potential is found to obey a modified PB equation, $\beta e \Delta V=\kappa^{2} \gamma(z) \sinh (\beta e V)$ 
where $\kappa^{2}=8 \pi \ell_{B} \rho_{s}^{\text {bulk }}$ is the Debye screening factor defined in terms of the bulk micro-ion concentration, and $\gamma(z)=\rho_{f}(z) / \rho_{f}$ is the normalized solvent density profile. In order to test the predictions of this approach, we have measured the fluid density profiles, $\rho_{f}(z)$, and solved Poisson's equation with the microionic densities given by Eq. (2), using Neumann boundary conditions, and assuming a smeared (uniform) surface charge on the wall. As shown in Fig. 1 this approach leads to results in very good agreement with the simulations profiles. Moreover, a further approximation can be proposed: The solution of the modified $\mathrm{PB}$ equation for electrostatic potential is actually very well approximated by the "bare" PB solution $V_{P B}(z)$ (corresponding to $\gamma(z)=1$ ), whose analytic expression can be found in the literature 11. This leads to $\rho_{ \pm}(z) \propto \rho_{f}(z) \exp \left[\mp \beta e V_{P B}(z)\right]$. The validity of this approximation -surprising in view of the strong layering effect at work- is emphasized in Fig. 11(inset), where the corresponding bare $\mathrm{PB}$ potential [1] is plotted against the "exact" electric potential. The latter is obtained from the simulations using Poisson's equation by integrating twice the charge density profile $\rho_{C}=e\left(\rho_{+}-\rho_{-}\right)$.

We now investigate the dynamical properties of the EDL. We first consider a streaming current experiment: an external volume force, $f_{0}$, is applied to the fluid in the $x$ direction, enforcing a Poiseuille flow in the cell, and the electric current, $I_{e}$, associated with the convective motion of the micro-ions is measured. The standard EDL description of this electrokinetic effect predicts a linear relationship between the current and the force, in the form [1] :

$$
I_{e}=-\frac{\epsilon_{d} \zeta}{\eta} \mathcal{A} f_{0}
$$

where $\eta$ is the shear viscosity of the fluid and $\mathcal{A}$ the fluid slab cross area. In the simulations, a force per particle is applied to all fluid particles and the corresponding electric current is measured. We emphasize that linear response (in the applied force) of the system was carefully checked. In the following we shall use this expression as the definition of the - apparent $-\zeta$ potential, in line with experimental procedures.

We first discuss the measured velocity profiles. The situation corresponding to a wetting substrate is shown in the main plot of Fig. 2 (here for $f_{0}=0.02$ in LJ units). The velocity profile is found to exhibit a parabolic shape as predicted by continuum hydrodynamics, even at the scale of the EDL. Moreover the viscosity, deduced from the curvature of the parabolic shape, is measured to keep its bulk value. Nevertheless, the no-slip BC is found to apply inside the liquid, at a distance of about one layer of solvent particle, in agreement with previous theoretical predictions [8]. This position of the no-slip BC here defines the "plane of shear" position, $z_{s}$, usually introduced in the electrokinetic literature 1]. As shown in Fig. 22 the layer of micro-ions located within $z_{s}$, does not contribute to the convective transport, thereby reducing the global streaming current. This immobile layer coincides with the so-called Stern layer of immobile micro-ions close to the charged surface [1]. Note moreover that we found $z_{s}$ to vary slightly with electrostatic parameters (surface charge $\Sigma$, Bjerrum length $\ell_{B}$ ), as expected.

On the other hand, the non-wetting case exhibits a very different behaviour, as shown in the inset of Fig. 2 First, concerning the velocity profile, a large amount of slip is found at the wall surface, in agreement with observations on uncharged non-wetting surfaces [8]. More quantitatively, slippage is characterized by a slip length, $b$, defined as the distance at which the linear extrapolation of the velocity profile vanishes. This amounts to replacing the no-slip BC by a partial slip BC, defined as $b \frac{\partial v}{\partial z}=v$ at the wall position [8]. As shown in the inset of Fig. 2] the velocity profile is found to be well fitted by the continuum hydrodynamics (parabolic) prediction, together with a partial slip BC, characterized by a non vanishing slip length (here $b \simeq 11 \sigma$ ). An important point here is that the first layer of micro-ions now contributes a large amount to the global streaming current in contrast to the wetting case, as emphasized in the inset of Fig. 2 The remobilization of the Stern layer therefore adds on to the slippage effect to increase the $\zeta$ potential.

We now summarize our results in Fig. 3 and plot the $\zeta$ potential [deduced from the measure of the charge current supplemented with Eq. [3] ] as a function of the Debye screening factor in the wetting and non-wetting

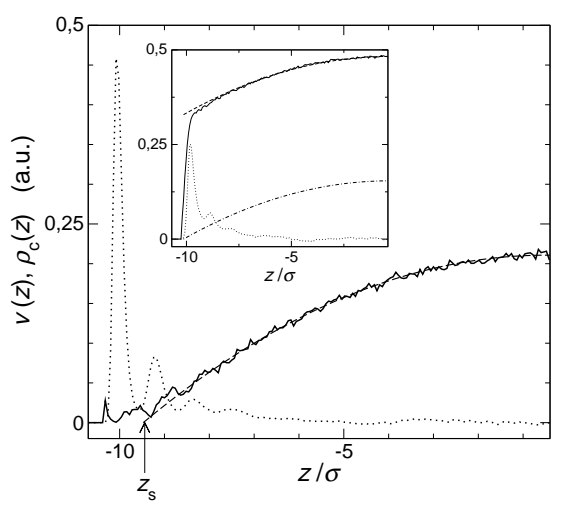

FIG. 2: Measured Poiseuille velocity profile (solid line) in the wetting case $\left(c_{F S}=1\right)$. Dashed line : hydrodynamic prediction using a no-slip BC at the 'plane of shear' located at $z_{s}$ (indicated by the arrow). To emphasize the existence of an immobile Stern layer, we also indicate the charge density profile $\rho_{C}(z)=e\left(\rho_{+}(z)-\rho_{-}(z)\right)$ (dotted line), with arbitrary units. The position of the wall (defined as that of the centers of the last layer of wall atoms) is at $z_{w}=-10.8 \sigma$. Inset: Results for the non-wetting case $\left(c_{F S}=0.5\right)$. Solid line : velocity profile measured in the simulation (shown on the same scale as in the main graph); Dashed line : hydrodynamic prediction with a partial slip BC, with a slip length $b=11 \sigma$; Dasheddot line : hydrodynamic prediction with a no-slip BC; Dotted line : charge density profile (arbitrary units). 
cases. In this figure the $\zeta$ potential is normalized by the bare surface potential $V_{0}$, obtained from the analytic PB expression 1] (see Fig. 11). Moreover, the slip length in the non-wetting case has been measured to barely depend on the screening factor, indicating that the micro-ions do not affect the fluid-solid friction. The overall conclusion

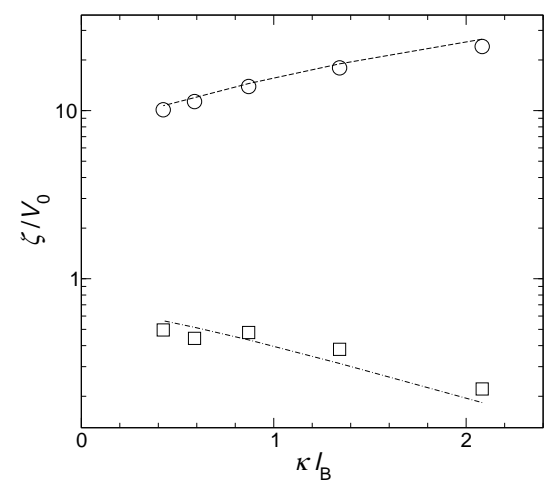

FIG. 3: The symbols show the $\zeta$ potential measured in MD as a function the screening factor $\kappa \ell_{B}$, for the wetting (bottom) and non-wetting substrate (top). The $\zeta$ potential is normalized by the bare surface potential $V_{0}$ obtained from the PB expression at a given $\kappa$ and surface charge (see the discussion on the inset of Fig. 1). For the wetting case (bottom), the dashed line is the PB electrostatic potential $V\left(z_{s}\right)$ where the 'plane of shear' position $z_{s}$ does not vary significantly with salt. For the non-wetting case (top), the dash-dotted line corresponds to the slip prediction, Eq. (4) (with $b=11 \sigma$ ).

from Fig. 3] is that non-wettability strongly amplifies the electrokinetic effects : the ratio between the $\zeta$ potential and the surface potential is much larger in the hydrophobic case as compared to the hydrophilic case. More precisely, in the wetting case the $\zeta$ potential is fixed by the electric properties of the surface, and coincides with the electric potential at the 'plane of shear', $\zeta \simeq V\left(z_{s}\right)$, as is usually assumed [1]. This is demonstrated in Fig. 3] where the simulation points for $\zeta$ are compared to the $\mathrm{PB}$ estimate for the electric potential, $V_{P B}\left(z_{s}\right)$, showing an overall very good agreement. Conversely, the $\zeta$ potential in the non-wetting case is dominated by the slip effect and the immobile Stern layer is completely absent. The effect of slip can be accounted for by considering the partial slip BC in the electrokinetic current $I_{e}=\int d S \rho_{C}(z) v(z)$ with $\rho_{C}(z)$ the charge density and $v(z)$ the velocity profile characterized by a slip length $b$. Within linearized PB description, and for the present planar geometry, the result for the current $I_{e}$, Eq. (3), may then be written $I_{e}=\frac{\epsilon_{d} V_{0}}{\eta}(1+\kappa b) f_{0}$ [2]. In the non-wetting case, this leads to

$$
\zeta=V_{0}(1+\kappa b)
$$

with $V_{0}$ the bare potential of the surface 13 . This expression is successfully compared in Fig. 3] to simulation results.
Finally we quote that we have tested a different electrokinetic geometry, corresponding to the more common electro-osmotic situation : flow response to the application of an electric field. The $\zeta$ potentials measured in this geometry (not shown here) are in full qualitative and quantitative agreement with the results in the present (streaming current) geometry, both on the wetting and non-wetting surfaces.

To conclude, we have shown using MD simulations that the notion of $\zeta$ potential, the cornerstone in the description of EDL dynamics, encompasses different physical mechanisms, depending on the wettability of the charged substrate. In the wetting situation, the $\zeta$ potential can be indeed directly related to surface charge properties, confirming hereby the traditional Stern layer picture [1]. In contrast, for non-wetting substrates, electric and slip effects are strongly intricated, leading for the $\zeta$ potential (Eq. 4) to an amplification ratio of $1+\kappa b$. Practically, this ratio can take large values, even for moderate slip lengths on the order of a few nanometers, since the Debye length $\kappa^{-1}$ usually lies in the nanometer range (to be specific, slip lengths of the order of tens of nanometers have been reported for hydrophobic silanized glass surfaces [10]). It would be moreover highly desirable to generalize Eq. (4) obtained for the planar case to other geometries (eg. spherical) where a new length (radius $R$ ) will compete with the debye $\left(\kappa^{-1}\right)$ and slip (b) lengths, Eq. (4) yielding the limiting behavior for $b / R, \kappa^{-1} / R \rightarrow 0$. This work also points to the difference between dynamical and static properties of the EDL, respectively characterized by the Zeta and bare surface potential, $\zeta$ and $V_{0}$. Independent static and dynamic measurements, using e.g. AFM or SFA, should therefore allow to probe the different mechanisms underlined in this study. Another route, with potential applications in micro-fluidics, consists in polarizing the solid substrate, i.e. "imposing" $V_{0}$, which should lead to large electrokinetic effects on non-wetting substrates.

* Electronic address: lbocquet@lpmcn.univ-lyon1.fr

[1] J. Lyklema, Foundations of Colloid and Interface Science, Vol II. (Academic Press, San Diego, 1995)

[2] H. Stone, A. Stroock, A. Ajdari, Ann. Rev. Flu. Mech. 36, 381 (2004)

[3] M. Bazant, T. Squires, Phys. Rev. Lett. 92066101 (2004)

[4] R. Netz Phys. Rev. Lett. 91138101 (2003)

[5] R. Qiao, N.R. Aluru Phys. Rev. Lett. 92198301 (2004)

[6] N.V. Churaev, J. Ralston, I.P. Sergeeva, V.D. Sobolev, Adv. Coll. Int. Sci. 96265 (2002)

[7] V. Marry, J.F. Dufrêche, M. Jardat, P. Turq, Mol. Phys. 101, 3111 (2003).

[8] J.-L. Barrat, L. Bocquet, Phys. Rev. Lett. 824671 (1999)

[9] S. Granick, H. Lee, Y. Zhu, Nature Mat. 2221 (2003)

[10] O. Vinogradova, Int. J. Miner. Process. 5631 (1999).

[11] We have used the MD code LAMMPS 2001, 
written by Steve Plimpton, available at http://www.cs.sandia.gov/ sjplimp/lammps.html

[12] S. Marcelja, Langmuir, 166081 (2000).
[13] For large $V_{0}$ the non-linear counterpart of this expression amounts to replacing $\kappa$ by $-\left.\partial_{z} V(z)\right|_{z=0} / V_{0}$ in Eq. (4). 